\title{
Laparoscopic Repair of a Subxiphoid Hernia After Omental Flap Reconstruction
}

\author{
Stephenie Poris, MD, Lauren Goldberg, MD, Jean Miner, MD \\ Center for Specialized Surgery, Florida Hospital, Orlando, Florida (all authors).
}

\begin{abstract}
Introduction: Hernias anterior to the sternum have rarely been reported and usually occur in the context of abdominal trauma. The literature reports hernia as a complication of omental flap reconstruction, but as yet there is little information on the laparoscopic repair of large subxiphoid hernia resulting from omental flap reconstruction.

Case: A 52-year-old woman presented 2 years after surgery with a large subxiphoid hernia containing the transverse colon. She had a history of metastatic follicular dendritic cell sarcoma after right lung lobectomy that led to infectious mediastinitis and a nonhealing sternal wound, for which she underwent omental flap repair.

Discussion: Our findings show the omentum can be reliably utilized to reconstruct thoracic defects. It can be a dependable salvage flap for previously failed reconstructions and can be transected in the face of a laparoscopic repair of a subxiphoid hernia. Furthermore, minimally invasive laparoscopic surgery provides a safe and effective surgical approach in a complicated patient with a subxiphoid hernia.
\end{abstract}

Key Words: Anterior wall reconstruction, Minimally invasive hernia repair, Omental flap, Subxiphoid hernia

Citation Poris S, Goldberg L, Miner J. Laparoscopic repair of a subxiphoid hernia after omental flap reconstruction. CRSLS e2015.00061. DOI: 10.4293/CRSLS.2015.00061.

Copyright (C) 2015 by SLS, Society of Laparoendoscopic Surgeons. This is an open-access article distributed under the terms of the Creative Commons Attribution-Noncommercial-ShareAlike 3.0 Unported license, which permits unrestricted noncommercial use, distribution, and reproduction in any medium, provided the original author and source are credited.

Address correspondence to: Stephenie Poris, MD, Center for Specialized Surgery, 2415 N Orange Avenue, Orlando, FL 32804. Telephone: 407-303-7399, Fax: 407-303-7305, E-mail: pollocksl@gmail.com

\section{INTRODUCTION}

Chest wall reconstruction is one of the most challenging procedures in cardiothoracic surgery and is usually necessary after tumor resection, infection, or radiation injuries. ${ }^{1}$ An omental flap has been used increasingly as either a primary repair or a secondary reinforcement salvage technique, because it provides great versatility in its angiogenic and immunogenic properties. ${ }^{2}$ Subxiphoid incisional hernia is rare, with an incidence ranging from 1 to $4.2 \%$, and it is notoriously difficult to repair and prone to recurrence. ${ }^{3,4}$ Although hernia is a well-documented complication of omental flap reconstruction, the surgical correction (especially minimally invasive) of a hernia of the magnitude and location of the subxiphoid incisional hernia has not been described.

\section{CASE}

Our patient was a 52-year-old Caucasian woman, with an extensive surgical history of 13 operations over a 1-year period for reconstruction of a nonhealing anterior chest wall defect associated with a metastatic follicular dendritic cell sarcoma. She presented to us with nausea and shortness of breath, and a computed tomographic (CT) scan demonstrated a $17.5 \times 5.6 \times 17.8$-cm subxiphoid hernia sac anterior to the sternum that contained the entire transverse colon.

Two years before, she had undergone pericardectomy for purulent pericarditis, and 3 months later, pectoralis major flaps were created for a nonhealing median sternotomy wound. The following month, percutaneous drains were placed for a persistent air leak from the right lung. A month later, laparoscopic mobilization of the omentum was performed, forming an omental flap affixed to the anterior chest wall with the pectoralis flaps overlying it to facilitate closure. She did well after the operation and was subsequently discharged with a stable chest wound (Figure 1).

On her recent presentation, the examination was remarkable for tenderness over an $8 \times 8$-cm subxiphoid defect, 

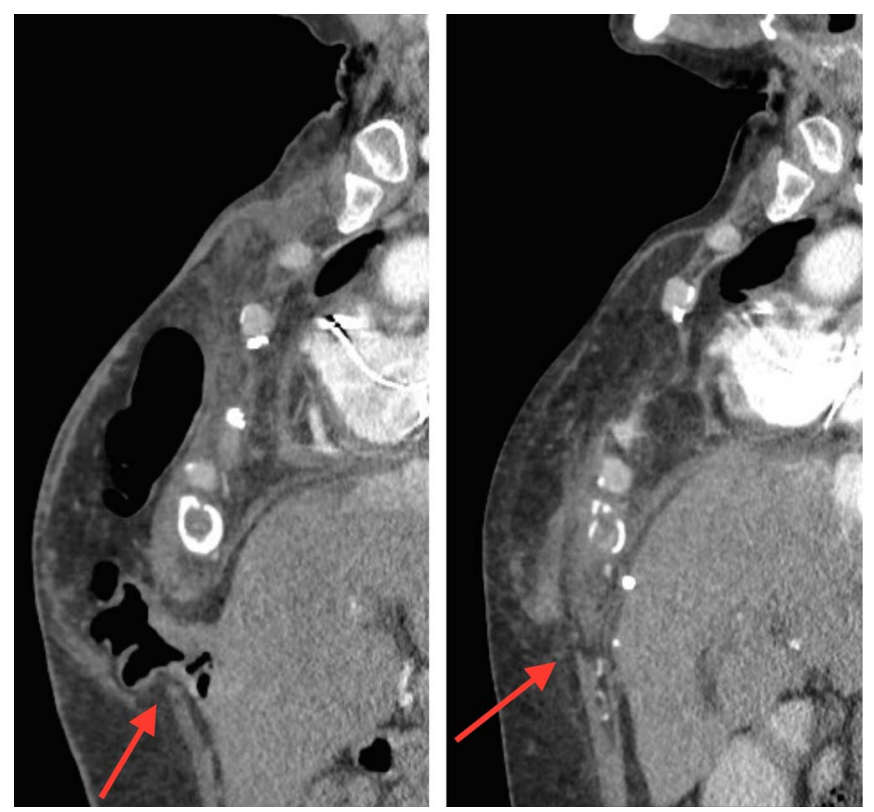

Figure 1. Left: Preoperative sagittal CT showing hernia defect inferior to the sternum and over the liver. Red arrow indicates defect with the transverse colon herniating through and over the anterior chest wall. Right: Post-operative CT at 5 months showing secured mesh over the anterior superior abdominal wall defect without reherniation.

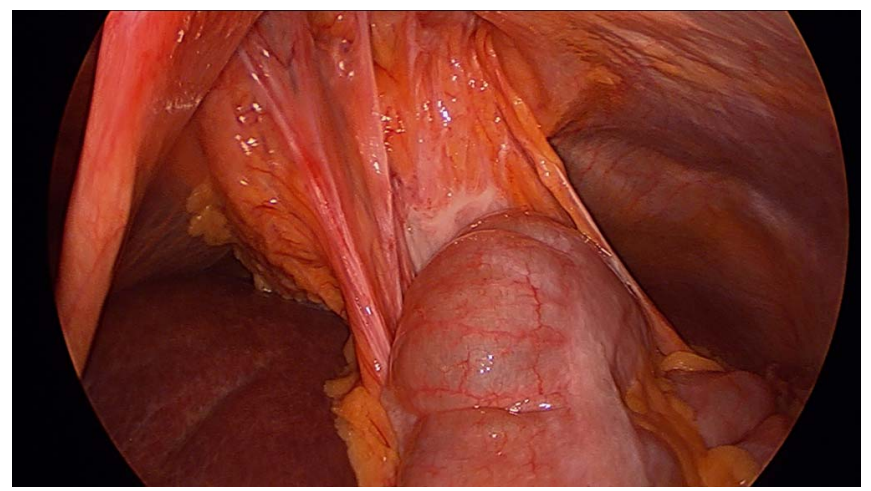

Figure 2. After abdominal insufflation: laparoscopic intraoperative view of the hernia sac fixed to the defect on the anterior abdominal wall. The transverse colon has partially receded from the chest into the abdomen.

with the contents extending from the epigastrium to the midchest. Audible bowel sounds were also appreciated. Labs were unremarkable, and bowel strangulation was ruled out. After cardiac and pulmonary optimization, she was taken to the operating room for repair. Based on prior research, we deemed that graft survival was likely after pedicle transection, secondary to collateral formation (Figure 2). After induction of anesthesia, an open Hassan

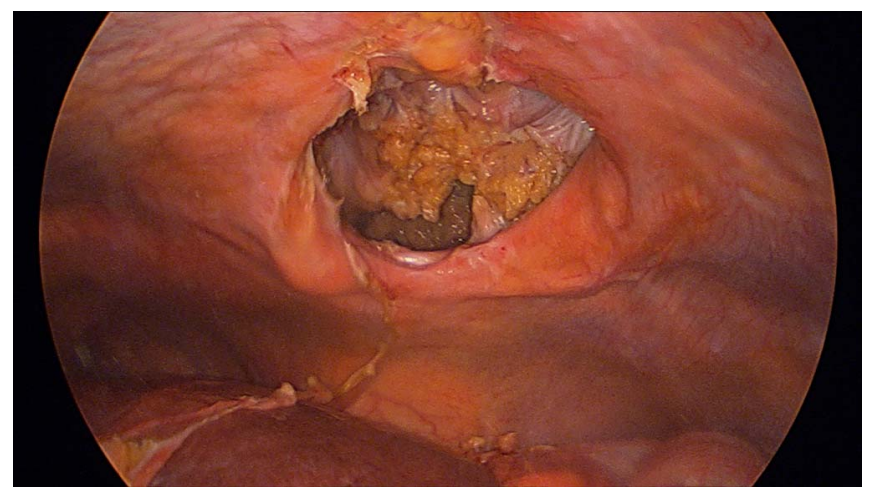

Figure 3. Laparoscopic intraoperative view of the defect after complete transection of the omental pedicle. All hernia contents have receded into the abdomen.

technique was used to enter the abdomen in the infraumbilical region. Two subsequent trocars were placed in the right and left midabdomen. Upon insufflation, the transverse colon spontaneously receded into the abdominal cavity. The omental pedicle and falciform ligament were ligated with an ultrasonic dissector at the level of the hernia, to facilitate adequate mesh placement (Figure 3). After transection of the omental pedicle, a $20-\mathrm{cm}$ duallayer monofilament polypropylene mesh was introduced with a hydrogel nonadherent barrier. The mesh was secured in an underlay fashion with a suture passer (Performance Medical, Inc., Minneapolis, Minnesota) used to place transfascial sutures through the abdominal wall. The inferior suture was placed in the supraumbilical region. Two additional sutures were placed just medial to the costal margin at the midpoint of the defect. A titanium tacker was used to secure the mesh approximately twothirds of the way superior to the abdominal wall, the entire inferior edge, and the right and left lateral edges inferior to the costal margin. Two additional transfascial sutures were placed in the right and left lateral aspects of the mesh. Because of the location of the defect and the proximity to the patient's heart and diaphragm, we believed that tacks and transfascial sutures could not be safely placed, and we therefore allowed for additional overlap of $8 \mathrm{~cm}$ superior to the uppermost aspect of the defect. Upon desufflation of the abdomen, the liver was noted to buttress the mesh, holding it in place (Figure 4).

The patient's postoperative recovery was unremarkable. She was started on a regular diet the following day and subsequently discharged home on postoperative day 4 . At the 6-week, 6-month, and 1-year follow-up appointments she was without evidence of hernia recurrence on physical examination and CT imaging. She was well from a surgical standpoint and was able to return to work. 


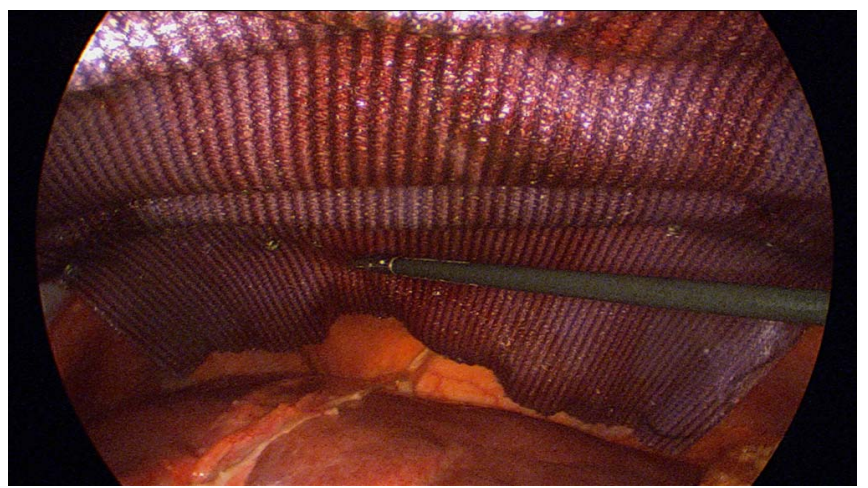

Figure 4. Laparoscopic view of the 20-cm dual-layer monofilament polypropylene mesh secured in an underlay fashion, with a hydrogel nonadherent barrier covering the hernia defect and extending approximately $8 \mathrm{~cm}$ from the superior aspect of the defect, for further overlap.

\section{DISCUSSION}

A subxiphoid hernia encompassing the entire transverse colon is described in another case report ${ }^{5}$; however, it was caused by blunt abdominal trauma that detached the diaphragm from the anterior portion of the abdominal wall. Most of the literature on omental flap reconstruction has reported diaphragmatic hernias, ${ }^{6}$ but our case involved a defect in the abdominal wall. Hernia formation is a known complication of omental flap reconstruction, but a subxiphoid hernia presents a special challenge because of its location and the surrounding anatomy. We preferred laparoscopic repair for our patient, whose body mass index of 43, significant comorbidities, and extensive surgical history involving a nonhealing sternal wound put her at significantly increased risk for surgical wound complications.

Chest wall reconstruction can be challenging, depending on location, size of the defect, and previous operations and radiotherapy. ${ }^{1}$ The omental flap has been increasingly used for diverse thoracic reconstruction because of its versatility, pliability, long pedicle length, and ease of harvest. ${ }^{1,4,7-9}$ The large surface area of well-vascularized tissue and rich lymphatic supply provides both angiogenic and immunogenic properties. ${ }^{4,8,9}$ In addition, the flap can provide adequate stability for respiration and water- and air-tight closure. ${ }^{1}$ However, a secondary flap revision procedure is not uncommon and has been reported in the literature as necessary to close the sternal wound. ${ }^{4}$

Over 25 years, Hultman et $\mathrm{al}^{2}$ studied the long-term sequelae of 60 patients with omental flaps ( 57 pedicled, 3 free) for a variety of thoracic wounds, to assess donor site morbidity, need for flap revision, complications, and spe- cific indications for use. The omentum was used as a primary flap in 39 patients and as a salvage flap in 21 . The leading morbidity was ventral hernia in 7 patients (11\%), all of whom underwent open herniorrhaphy. The authors described the division of the pedicle without flap necrosis. In a more recent study, Tukiainen ${ }^{1}$ also demonstrated that the flap can be safely divided 6 to 12 months after reconstruction, to close the abdominal fascia layer. In our case, we observed that after transection of the flap, there was adequate blood flow with no postoperative necrosis or other complication.

Although the origin is not fully known, subxiphoid incisional hernia is thought to be caused by fascial dehiscence in the presence of weakened soft tissues in the xiphoid region. ${ }^{2,10,11}$ Over time, hernia formation is possible because repetitive breathing and coughing create lateral traction forces exerted by abdominal and transverse thoracic muscles, fibers of the linea alba, costoxiphoid ligaments, and the diaphragm. ${ }^{3}$ Repair is complicated when cartilaginous or osseous structures border the fascial defect, most notoriously at the xiphoid and costal margins after sternotomy. ${ }^{3}$ Because of the nature of this difficult repair, a laparoscopic approach may provide better visualization of the defect and allow for more extensive repair.

Laparoscopic treatment of subxiphoid hernias was first performed in 2000 and has been widely adopted, as it can provide minimal perioperative morbidity and expedite functional recovery. ${ }^{12}$ It is especially imperative to ensure a safe repair for complex hernias in obese patients, whose multiple comorbidities and defects in tissue structure and healing predispose them to perioperative complications. ${ }^{12}$ Laparoscopic repair of subxiphoid incisional hernia is regarded as the approach of choice for obese patients with a BMI $>30 .{ }^{12}$ Attention to technical details, including ensuring an adequate overlap, careful adhesiolysis, and correct fixation of the prosthesis is recommended. ${ }^{13}$ Complications and recurrences have been found to be less frequent than those that occur after an open approach. ${ }^{13}$ In other studies of open versus laparoscopic approaches, research has established less intraoperative blood loss, morbidity, wound complications, and decreased hospital length of stay with the minimally invasive technique. ${ }^{13,14-16}$

It is imperative to the success of an upper abdominal operation, especially in high-risk patients, to optimize the patient's respiratory status. The laparoscopic technique enables early ambulation to prevent further pulmonary complications. Our patient was very susceptible to postoperative pulmonary complication, as her pulmonary function was already dramatically reduced by a lobec- 
tomy. Wound complications also posed a major concern, with her history of radiation and a refractory suppurative sternal wound. Any perioperative complications, such as seroma, hematoma, and pain at transabdominal suture sites, are generally less numerous and easier to manage. ${ }^{13,15}$ Laparoscopy offers a reduced rate of hernia recurrence, which is important, as it decreases the necessity of further operations in high-risk patients. ${ }^{15,16}$

\section{CONCLUSIONS}

In planning this surgery, many factors had to be taken into consideration. Not only did the patient have an extensive history of more than 13 thoracic surgeries, complicated by infections, long intensive care unit stays, and a nonhealing sternal wound, but she also had undergone chest radiation. We found that the omentum can be reliably used in the reconstruction needed to repair thoracic defects. It can be a dependable salvage flap for previously failed reconstructions and can be transected in a laparoscopic repair of a subxiphoid hernia. Secondary to our patient's multiple comorbidities, careful planning of the surgery and a minimally invasive laparoscopic technique were used for successful reduction of the transverse colon into the abdomen and closure of the hernia defect distal to the xiphoid process.

\section{References:}

1. Tukiainen E. Chest wall reconstruction after oncological resections. Scand J Surg. 2013;102:9-13.

2. Hultman CS, Culbertson JH, Jones GE, et al. Thoracic reconstruction with the omentum: indications, complications, and results. Ann Plast Surg. 2001;46:242-249.

3. Kim H, Kim K, Hwang HY, et al. Subxiphoid incisional hernia development after coronary artery bypass grafting. Korean J Thorac Cariovasc Surg. 2012;45:161-165.

4. Losanoff JE, Basson MD, Laker S, Weiner M, Webber JD, Gruber SA. Subxiphoid incisional hernias after median sternotomy. Hernia. 2007;11:473-479.

5. McBeth PB, Widder S, McColl RJ, et al. Traumatic abdominal hernia with presternal herniation. Can J Surg. 2009;52:E207E208.
6. Muysoms FE, Cathenis KK, Hamerlijnck RP, Claeys DA. Laparoscopic repair of iatrogenic diaphragmatic hernias after sternectomy and pedicled omentoplasty. Hernia. 2009;13:617-623.

7. Ghazi BH, Carlson GW, Losken A. Use of the greater omentum for reconstruction of infected sternotomy wounds: a prognostic indicator. Ann Plast Surg. 2008;60:169-173.

8. Hultman CS, Carlson GW, Losken A, et al. Utility of the omentum in the reconstruction of complex extraperitoneal wounds and defects: donor-site complications in 135 patients from 1975 to 2000. Ann Surg. 2002;235:782-795.

9. Vyas RM, Prsic A, Orgill DP. Transdiaphragmatic omental harvest: a simple efficient method of sternal wound coverage. Plastic Reconstr Surg. 2013;131:544.

10. Murari VJ, Alexander GL, Cassivi SD. Massive intrapericardial herniation of stomach following pericardial window. Hernia. 2004;8:273-276.

11. Conze J, Prescher A, Kisielinski K, Klinge U, Schumpelick V. Technical consideration for subxiphoidal incisional hernia repair. Hernia. 2005;9:84-87.

12. Novitsky YW, Cobb WS, Kercher KW, Matthews BD, Sing RF, Heniford BT. Laparoscopic ventral hernia repair in obese patients: a new standard of care. Arch Surg. 2006;141:57-61.

13. Cuccurullo D, Piccoli M, Agresta F. Laparoscopic ventral incisional hernia repair: evidence-based guidelines of the first Italian Consensus Conference. Hernia. 2013;17;557-566.

14. Bingener J, Buck L, Richards M, et al. Long-term outcomes in laparoscopic versus open ventral hernia repair. Arch Surg. 2007; 142:562-567.

15. Chelala E, Debardemaeker Y, Elias B, Charara F, Dessily M, Allé JL. Eighty-five redo surgeries after 733 laparoscopic treatments for ventral and incisional hernia: adhesion and recurrence analysis. Hernia. 14:123-129, 2010.

16. Cobb WS, Kercher KW, Matthews BD, et al. Laparoscopic ventral hernia repair: a single center experience. Hernia. 2006; 10:236-242.

17. Landau O, Raziel A, Matz A, Kyzer S, Haruzi I. Laparoscopic repair of poststernotomy subxiphoid epigastric hernia. Surg Endosc. 2001;15:1313-1314. 